PROCEEDINGS OF THE

AMERICAN MATHEMATICAL SOCIETY

Volume 131, Number 8, Pages 2515-2525

S 0002-9939(02)06832-6

Article electronically published on November 14, 2002

\title{
SOME REMARKS ON SPREADING MODELS AND MIXED TSIRELSON SPACES
}

\author{
A. MANOUSSAKIS
}

(Communicated by N. Tomczak-Jaegermann)

\begin{abstract}
We prove that if a Banach space with a bimonotone shrinking basis does not contain $\ell_{1}^{\omega}$ spreading models but every block sequence of the basis contains a further block sequence which is a $c-\ell_{1}^{n}$ spreading model for every $n \in \mathbb{N}$, then every subspace has a further subspace which is arbitrarily distortable. We also prove that a mixed Tsirelson space $T\left[\left(\mathcal{S}_{n}, \theta_{n}\right)_{n}\right]$, such that $\theta_{n} \searrow 0$, does not contain $\ell_{1}^{\omega 2}$ spreading models.
\end{abstract}

\section{INTRODUCTION}

A Banach space $X$ with a basis $\left(e_{i}\right)$ is an asymptotic $\ell_{1}$ space if there exists $c>0$ such that for all $n$ and all $e_{n}<x_{1}<\ldots<x_{n}$,

$$
\left\|\sum_{i=1}^{n} x_{i}\right\| \geq c \sum_{i=1}^{n}\left\|x_{i}\right\|
$$

The first non-trivial example of an asymptotic $\ell_{1}$ space was discovered by Tsirelson [17]. Recent results [6], [7], [15], have shown the necessity of studying the higher ordinal structure of an asymptotic $\ell_{1}$ Banach space in order to obtain results on the global structure of its infinite dimensional subspaces. A normalized sequence $\left(x_{n}\right)_{n}$ in a Banach space $X$ is said to be a $c-\ell_{1}^{\xi}$ spreading model if

$$
\left\|\sum_{n \in F} \alpha_{n} x_{n}\right\| \geq c \sum_{n \in F}\left|\alpha_{n}\right| \forall F \in \mathcal{S}_{\xi},\left(a_{n}\right)_{n \in F} \subset \mathbb{R},
$$

where $\mathcal{S}_{\xi}, \xi<\omega_{1}$, are the generalized Schreier families defined in [1].

It is well known that if a separable Banach space $X$ does not contain $\ell_{1}$, then there exists $\xi<\omega_{1}$, such that $X$ does not contain an $\ell_{1}^{\xi}$ spreading model. A complete classification of normalized weakly null sequences, in connection with spreading models, has been provided in [7].

Spreading models is a basic tool for the study of the asymptotic structure of a Banach space. The structure of the spreading models may even determine the geometry of the space [14]. Spreading models have been employed in [10] to prove the existence of strictly singular non-compact operators in certain Hereditarily Indecomposable mixed Tsirelson spaces.

Received by the editors November 13, 2001 and, in revised form, March 24, 2002.

2000 Mathematics Subject Classification. Primary 46B03, 46B20, 46 B45.

Key words and phrases. Schreier families, spreading model.

(C)2002 American Mathematical Society 
The idea of investigating the geometry of a Banach space by studying its asymptotic finite-dimensional subspaces emerged naturally in recent studies related to problems of distortion, i.e. the stabilization of equivalent norms on infinitedimensional subspaces [2], [3], [5], [9], 15].

A Banach space $(X,\|\cdot\|)$ is said to be $\lambda$-distortable if there exists an equivalent norm $|\cdot|$ on $X$ so that

$$
\inf _{Y} \sup \left\{\frac{|x|}{|y|}: x, y \in S(Y,\|\cdot\|)\right\} \geq \lambda,
$$

where the infimum is taken over all infinite-dimensional subspaces $Y$ of $X . X$ is arbitrarily distortable if it is $\lambda$-distortable for all $\lambda>1$. In section 2 we prove the following:

Theorem. Let $X$ be a Banach space with a bimonotone shrinking basis $\left(e_{i}\right)$ such that

(1) $X$ does not contain an $\ell_{1}^{\omega}$ spreading model.

(2) For every $n \in \mathbb{N}$, every block sequence of $\left(e_{i}\right)$ contains a further block sequence which is a $c-\ell_{1}^{n}$ spreading model.

Then every subspace of $X$ contains a further subspace which is arbitrarily distortable.

The relation of the distortion problem with spreading models has been studied in [15], 9]. In [9] a criterion has been provided related to $\ell_{1}^{n}$ spreading models, which implies the distortion of certain asymptotic $\ell_{1}$ Banach spaces. The motivation for this theorem was the space constructed in [5], where an example of a mixed Tsirelson space $X$ was given which has $c-\ell_{1}^{n}$ spreading models in every block subspace but has no $\ell_{1}^{\omega}$ spreading model. The norm of $X$ satisfies, for an appropriate sequence $\left(k_{j}, \theta_{j}\right)$, the implicit equation

$$
\|x\|=\max \left\{\|x\|_{\infty}, \sup \left\{\sum_{k=1}^{n}\left\|\left.x\right|_{[n,+\infty)}\right\|_{j_{k}}: n \in \mathbb{N}, j_{1}<j_{2}<\ldots<j_{n}\right\}\right\},
$$

where $\|x\|_{j}=\sup \left\{\theta_{j} \sum_{i \in F}\left\|E_{i} x\right\|:\left(E_{i} x\right)_{i \in F} \mathcal{S}_{k_{j}}\right.$-admissible $\}$.

To prove the theorem we use some results proved by E.Odell, N.TomczakJaegermann and R.Wagner 15. In this paper, for a Banach space $X$ with basis $\left(e_{i}\right)$, certain indices $\left(\delta_{\alpha}\left(x_{i}\right)\right)_{\alpha<\omega_{1}}$, for a block sequence $\left(x_{i}\right)$, have been introduced and studied. Roughly speaking, the indices $\left(\delta_{\alpha}\left(x_{i}\right)\right)_{\alpha<\omega_{1}}$ measure the strong presence of $\ell_{1}$ in the subspace $\left\langle\left(x_{i}\right)\right\rangle$ in connection with the families $\left(\mathcal{S}_{\alpha}\right)_{\alpha<\omega_{1}}$. The notion of $\Delta$-spectrum, $\Delta(X)$, is also introduced. Roughly, $\Delta(X)$ is the set of all $\gamma=\left(\gamma_{\alpha}\right)_{\alpha<\omega_{1}}$, where $\gamma_{\alpha}$ is the stabilization of $\delta_{\alpha}\left(y_{i}\right)$ for some block basis $\left(y_{i}\right)$ of $\left(e_{i}\right)$. Using the stabilization result from [15], we prove that every block subspace has a further subspace $Y$ such that: For every $n \in \mathbb{N}$ there exist two asymptotic sets $A_{n}$ and $B_{n}$ in $Y$ and a subset $A_{n}^{*}$ of $X^{*}$ such that the equivalent norm

$$
\||x|\|=\gamma_{n}\|x\|+\sup \left\{x^{*}(x): x^{*} \in A_{n}^{*}\right\}
$$

is a $\approx \frac{1}{\gamma_{n}}$ distortion for $Y$. Since $\gamma_{\omega}=0$, from the continuity of the indices $\left(\gamma_{\alpha}\right)_{\alpha<\omega_{1}}$ [15], we have that $Y$ is arbitrarily distortable.

In section[3] we prove that in the mixed Tsirelson spaces $T\left[\left(\mathcal{S}_{n}, \theta_{n}\right)_{n}\right]$ the following holds. 
Theorem. Let $X=T\left[\left(\mathcal{S}_{n}, \theta_{n}\right)_{n}\right]$ such that $\theta_{n+m} \geq \theta_{n} \theta_{m}, \lim _{n} \theta_{n}=0$. Then the space $X$ does not contain $\ell_{1}^{\omega 2}$ spreading models.

In [5] it has been proved that, assuming $\lim _{n} \theta_{n}^{1 / n}=1$, the space $T\left[\left(\mathcal{S}_{n}, \theta_{n}\right)_{n}\right]$ contains an $\ell_{1}^{\omega}$ spreading model. The existence of $\ell_{1}^{\omega}$ spreading models in these spaces is based on the disjoint representability of $c_{0}$ in these spaces [4]. Another approach to the existence of $\ell_{1}^{\omega}$ in certain mixed Tsirelson spaces has been provided in [10. The key point for the proof of this theorem is to produce for every normalized block sequence $\left(x_{n}\right)$ of the basis, a vector in the linear span of $\left(x_{n}\right)$, whose norm is arbitrarily small yet its support with respect to $\left(x_{n}\right)$ belongs to $\mathcal{S}_{\omega 2}$. The dual of the original Tsirelson's space [17] contains no $\ell_{1}^{\omega}$ spreading model. This is due to the fact that every block sequence is equivalent to a subsequence of the basis.

\section{Preliminaries}

Notation. Let $\left(e_{i}\right)_{i=1}^{\infty}$ be a basic sequence. For $x=\sum_{i=1}^{\infty} a_{i} e_{i}$ the support of $x$ w.r.t. $\left(e_{i}\right)$ is the set $\operatorname{supp} x=\left\{i \in \mathbb{N}: a_{i} \neq 0\right\}$. The range of $x$, written range $(x)$, is the smallest interval of $\mathbb{N}$ containing the support of $x$. For finite subsets $E, F$ of $\mathbb{N}$, $E<F$ means $\max E<\min F$ or either $E$ or $F$ is empty. For $n \in \mathbb{N}, E \subset \mathbb{N}, n<E$ (resp. $E<n$ ) means $n<\min E$ (resp. $\max E<n$ ). For $x, y$ in $c_{00}, x<y$ means $\operatorname{supp} x<\operatorname{supp} y$. For $n \in \mathbb{N}, x \in c_{00}$, we write $n<x($ resp. $x<n)$ if $n<\operatorname{supp} x$ (resp. $\operatorname{supp} x<n)$. We say that the sets $E_{i} \subset \mathbb{N}, i=1, \ldots, n$, are successive if $E_{1}<E_{2}<\ldots<E_{n}$. Similarly, the vectors $x_{i}, i=1, \ldots n$, are successive if $x_{1}<x_{2}<\ldots<x_{n}$. If $\left(x_{i}\right)$ is a block sequence of $\left(e_{i}\right)$ we write $\left(x_{i}\right) \prec\left(e_{i}\right)$. For $x=\sum_{i=1}^{\infty} a_{i} e_{i}$ and $E$ a subset of $\mathbb{N}$, we denote by $E x$ the vector $E x=\sum_{i \in E} a_{i} e_{i}$. For an infinite subset $M$ of $\mathbb{N}$ we denote by $[M]$ the class of infinite subsets of $M$ and by $[M]^{<\omega}$ the class of finite subsets of $M$.

The generalized Schreier families $\left\{\mathcal{S}_{\xi}\right\}_{\xi<\omega_{1}}$, introduced in [1], are defined by transfinite induction as follows:

$$
\mathcal{S}_{0}=\{\{n\}: n \in \mathbb{N}\} \cup\{\emptyset\} .
$$

Suppose that the families $\mathcal{S}_{\alpha}$ have been defined for all $\alpha<\xi$.

If $\xi=\zeta+1$, we set

$\mathcal{S}_{\xi}=\left\{F \in[\mathbb{N}]^{<\omega}: F=\bigcup_{i=1}^{n} F_{i}, n \in \mathbb{N}, \forall i \leq n F_{i} \in \mathcal{S}_{\zeta}\right.$ and $\left.n \leq F_{1}<\ldots<F_{n}\right\} \cup\{\emptyset\}$.

If $\xi$ is a limit ordinal, let $\left(\xi_{n}+1\right)_{n}$ be a sequence of successor ordinals which strictly increases to $\xi$. We set

$$
\mathcal{S}_{\xi}=\left\{F \in \mathbb{N}^{<\omega}: \text { for } \quad \text { some } n \in \mathbb{N}, n \leq \min F \text { and } F \in \mathcal{S}_{\xi_{n}+1}\right\} .
$$

If $N=\left(n_{i}\right)_{i}$ is an infinite subset of $\mathbb{N}$, then we define

$$
\mathcal{S}_{\xi}[N]=\left\{F: F \subset N, F \in \mathcal{S}_{\xi}\right\} \text { and } \mathcal{S}_{\xi}(N)=\left\{\left(n_{i}\right)_{i \in F}: F \in \mathcal{S}_{\xi}\right\} .
$$

Proposition 1.1. (a) 2] Let $N \in[\mathbb{N}]$. Then there exists $L=\left(\ell_{i}\right) \in[N]$ so that for all $\alpha<\omega_{1}$,

$$
\left(\ell_{i}\right)_{i \in F} \in \mathcal{S}_{\alpha} \Rightarrow\left(\ell_{i}\right)_{i \in F \backslash(\min F)} \in \mathcal{S}_{\alpha}(N) .
$$

(b) [15] Let $\beta<\alpha<\omega_{1}$. There exists $n_{0} \in \mathbb{N}$ such that

$$
n_{0}<F \in \mathcal{S}_{\beta} \Rightarrow F \in \mathcal{S}_{\alpha} .
$$

(c) [15] Let $\beta<\alpha<\omega_{1}$. There exists $M \in[\mathbb{N}]$ such that $\mathcal{S}_{\alpha}\left[\mathcal{S}_{\beta}\right](M) \subset \mathcal{S}_{\beta+\alpha}$. 
We next pass to the definition of the repeated averages hierarchy introduced in 7]. We let $\left(e_{n}\right)$ denote the standard basis of $c_{00}$. For every countable ordinal $\xi$ and every $M \in[\mathbb{N}]$, we define a convex block sequence $\left(\xi_{n}^{M}\right)_{n=1}^{\infty}$ of $\left(e_{n}\right)$ by transfinite induction on $\xi$ in the following manner:

If $\xi=0$ and $M=\left(m_{n}\right)_{n=1}^{\infty}$, then $\xi_{n}^{M}=e_{m_{n}}$, for all $n \in \mathbb{N}$.

Assume that $\left(\zeta_{n}^{M}\right)_{n=1}^{\infty}$ has been defined for all $\zeta<\xi$ and $M \in[\mathbb{N}]$. Let $\xi=\zeta+1$. We set

$$
\xi_{1}^{M}=\frac{1}{m_{1}} \sum_{i=1}^{m_{1}} \zeta_{i}^{M}
$$

where $m_{1}=\min M$. Suppose that $\xi_{1}^{M}<\ldots<\xi_{n}^{M}$ have been defined. Let

$$
M_{n}=\left\{m \in M: m>\max \operatorname{supp} \xi_{n}^{M}\right\} \quad \text { and } \quad k_{n}=\min M_{n} .
$$

Set

$$
\xi_{n+1}^{M}=\frac{1}{k_{n}} \sum_{i=1}^{k_{n}} \zeta_{i}^{M_{n}}=\xi_{1}^{M_{n}} .
$$

If $\xi$ is a limit ordinal, let $\left(\xi_{n}+1\right)_{n}$ be the sequence of ordinals associated to $\xi$, and also let $M \in[\mathbb{N}]$. Define

$$
\xi_{1}^{M}=\left[\xi_{m_{1}}+1\right]_{1}^{M}
$$

where $m_{1}=\min M$. Suppose that $\xi_{1}^{M}<\ldots<\xi_{n}^{M}$ have been defined. Let

$$
M_{n}=\left\{m \in M: m>\max \operatorname{supp} \xi_{n}^{M}\right\} \text { and } k_{n}=\min M_{n} .
$$

Set

$$
\xi_{n+1}^{M}=\left[\xi_{k_{n}}+1\right]_{1}^{M_{n}} .
$$

The inductive definition of $\left(\xi_{n}^{M}\right)_{n=1}^{\infty}, M \in[\mathbb{N}]$, is now complete. We note that $\operatorname{supp} \xi_{n}^{M} \in \mathcal{S}_{\xi}$, for all $M \in[\mathbb{N}], \xi<\omega_{1}$ and $n \in \mathbb{N}$.

Definition 1.2. (a) Let $k \in \mathbb{N}$. A finite sequence $\left(E_{i}\right)_{i=1}^{m}$ of successive subsets of $\mathbb{N}$ is said to be $\mathcal{S}_{k}$-admissible if $\left\{\min E_{i}\right\}_{i=1}^{m} \in \mathcal{S}_{k}$. A finite block sequence $\left(x_{i}\right)_{i=1}^{m}$ in $c_{00}$ is said to be $\mathcal{S}_{k}$-admissible if $\left(\operatorname{supp} x_{i}\right)_{i=1}^{m}$ is $\mathcal{S}_{k}$-admissible. If $\left(y_{i}\right)$ is a block sequence of the basis of $c_{00},\left(x_{i}\right)_{i}$ is a block sequence of $\left(y_{i}\right)$, and $\widetilde{E}_{i}$ is the range of $x_{i}$ with respect to (w.r.t.) the basic sequence $\left(y_{i}\right)$, then the block sequence $\left(x_{i}\right)$ is $\mathcal{S}_{n}$-admissible w.r.t. $\left(y_{i}\right)$ if $\left\{\min \widetilde{E}_{i}\right\}_{i} \in \mathcal{S}_{n}$.

(b) Let $\left\{k_{n}\right\}_{n}$ be an increasing sequence of integers and $\left\{\theta_{n}\right\} \subset(0,1)$ such that $\theta_{n} \searrow 0$. The mixed Tsirelson space $X=T\left[\left(\mathcal{S}_{k_{n}}, \theta_{n}\right)_{n=1}^{\infty}\right]$ is the completion of $c_{00}$ under the norm which satisfies the implicit equation

$$
\|x\|=\max \left\{\|x\|_{\infty}, \sup _{n} \theta_{n}\left\{\sup \sum_{i=1}^{m}\left\|E_{i}(x)\right\|\right\}\right\},
$$

where the inside supremum is taken over all $\mathcal{S}_{k_{n}}$-admissible families $\left\{E_{i}\right\}_{i=1}^{m}, m \in \mathbb{N}$. The space $X$ is a reflexive Banach space, and the sequence $\left(e_{i}\right)$ is a basis on $X$.

An essential role in our proofs is played by the following special vectors.

Definition 1.3. Let $\left(x_{n}\right)$ be a normalized block sequence of $\left(e_{n}\right), \varepsilon>0$ and $\zeta<\xi<\omega_{1}$. Set $m_{n}=\min \operatorname{supp} x_{n}$ and $M=\left(m_{n}\right)_{n}$. 
An $(\varepsilon, \xi, \zeta)$ basic special convex combination (basic s.c.c.) for $M$ is any vector of the form $\xi_{1}^{L}=\sum_{n} \xi_{1}^{L}\left(m_{n}\right) e_{m_{n}}, L \in[M]$ and $\left\|\xi_{1}^{L}\right\|_{\zeta}<\varepsilon$, where $\left\|\sum \alpha_{i} e_{i}\right\|_{\zeta}=$ $\sup \left\{\sum_{i \in F}\left|\alpha_{i}\right|: F \in \mathcal{S}_{\zeta}\right\}$.

An $(\varepsilon, \xi, \zeta)$ special convex combination, (s.c.c.), of $\left(x_{n}\right)$ is any vector of the form $\sum_{n} \xi_{1}^{L}\left(m_{n}\right) x_{n}$, such that $\sum_{n} \xi_{1}^{L}\left(m_{n}\right) e_{m_{n}}$ is an $(\varepsilon, \xi, \zeta)$-basic s.c.c.

Proposition $1.4([6])$. For every $M \in[\mathbb{N}], \varepsilon>0$ and all ordinals $\zeta<\xi<\omega_{1}$, there exists $N \in[M]$ such that $\left\|\xi_{1}^{L}\right\|_{\zeta}<\varepsilon$ for all $L \in[N]$.

It is not hard to see that the average $n_{1}^{L}$ is a $\left(\frac{3}{\min L}, n, n-1\right)$-basic s.c.c. for every $L \in[M]$.

Definition 1.5. Let $\xi<\omega_{1}$ and $\delta>0$. A normalized sequence $\left(x_{n}\right)$ in a Banach space is an $\delta-\ell_{1}^{\xi}$ spreading model if

$$
\left\|\sum_{i \in F} \alpha_{i} x_{i}\right\| \geq \delta \sum_{i \in F}\left|\alpha_{i}\right|
$$

for every $F \in \mathcal{S}_{\xi}$ and all choices of scalars $\left(\alpha_{i}\right)_{i \in F}$.

$\left(x_{n}\right)$ is called an $\ell_{1}^{\xi}$ spreading model if it is an $\delta-\ell_{1}^{\xi}$ spreading model for some $\delta>0$.

2.

Theorem 2.1. Let $X$ be a Banach space with a bimonotone shrinking basis $\left(e_{i}\right)$ such that

(1) $X$ does not contain an $\ell_{1}^{\omega}$ spreading model.

(2) There exists $c>0$ such that for every $n \in \mathbb{N}$, every block sequence of $\left(e_{i}\right)$ contains a further block sequence which is a $c-\ell_{1}^{n}$ spreading model.

Then every subspace of $X$ contains a further subspace which is arbitrarily distortable.

Our proof is based on the following results of E.Odell, N. Tomczak-Jaegermann and R.Wagner [15].

Definition $2.2([15])$. Let $\mathcal{F}$ be a regular set of finite subsets of $\mathbb{N}$. For a basic sequence $\left(x_{i}\right)_{i}$ in $X$ we define

$$
\begin{aligned}
\delta_{\mathcal{F}}=\sup \left\{\delta \geq 0:\left\|\sum_{i=1}^{k} y_{i}\right\| \geq\right. & \delta \sum_{i=1}^{k}\left\|y_{i}\right\| \text { whenever }\left(y_{i}\right)_{1}^{k} \prec\left(x_{i}\right) \\
& \text { is } \left.\mathcal{F} \text {-admissible w.r.t. }\left(x_{i}\right)\right\} .
\end{aligned}
$$

For $\alpha<\omega_{1}$ we set $\delta_{\alpha}\left(x_{i}\right)=\delta_{\mathcal{S}_{\alpha}}\left(x_{i}\right)$ and $\delta_{\alpha}(X)=\delta_{\mathcal{S}_{\alpha}}(X)$.

Definition $2.3([15])$. Let $X$ be a Banach space with basis $\left(e_{i}\right)$, and let $\gamma=$ $\left(\gamma_{\alpha}\right)_{\alpha<\omega_{1}} \subset \mathbb{R}$. We say that a basic sequence $\left(x_{i}\right)$ in $X \Delta$-stabilizes $\gamma$, if there exists $\varepsilon_{n} \searrow 0$ so that for every $\alpha<\omega_{1}$ there exists $m \in \mathbb{N}$ such that for every $n \geq m$ if $\left(y_{i}\right) \prec\left(x_{i}\right)_{n}^{\infty}$, then $\left|\delta_{\alpha}\left(y_{i}\right)-\gamma_{\alpha}\right|<\varepsilon_{n}$.

The $\Delta$-spectrum of $X, \Delta(X)$, is defined to be the set of all $\gamma$ 's so that there exists $\left(x_{i}\right) \prec\left(e_{i}\right)$ such that $\left(x_{i}\right) \Delta$-stabilizes $\gamma$.

Proposition 2.4 ([15, Proposition 4.11]). (1) Let $X$ be a Banach space with a basis $\left(e_{i}\right)$. Then there exists $\gamma=\left(\gamma_{\alpha}\right)_{\alpha<\omega_{1}}$ and $\left(x_{i}\right)$ block sequence of $\left(e_{i}\right)$ so that $\left(x_{i}\right) \Delta$-stabilizes $\gamma$.

(2) $\left(\gamma_{\alpha}\right)_{\alpha<\omega_{1}}$ is a continuous function of $\alpha$. 
In the above definitions, the admissibility refers with respect to the block basis $\left(x_{i}\right)$ itself. It has been proved in [2] that, if we consider reference level for admissibility fixed the basis, then these two concepts of spectrum actually coincide.

Proof of Theorem 2.1. Let $W$ be a block subspace of $X$. Then there exists a block sequence $\left(y_{i}\right)_{i}$ in $W$ and $\left(\gamma_{\alpha}\right)_{\alpha<\omega_{1}}$ such that $\left(y_{i}\right) \Delta$-stabilizes $\left(\gamma_{\alpha}\right)_{\alpha<\omega_{1}}$. Let $\varepsilon_{n} \searrow 0$ be the sequence in the stabilization of $\left(\gamma_{\alpha}\right)_{\alpha<\omega_{1}}$ by $\left(y_{i}\right)$, i.e. for every $\alpha<\omega_{1}$ there exists $m \in \mathbb{N}$ such that for every $n \geq m$ if $\left(x_{i}\right) \prec\left(y_{i}\right)_{n}^{\infty}$, then $\left|\delta_{\alpha}\left(x_{i}\right)-\gamma_{\alpha}\right|<\varepsilon_{n}$.

Inductively choose a strictly increasing sequence $(m(n))_{n}$ of integers such that $\varepsilon_{m(n)}<\frac{\left(\delta_{1}\left(y_{i}\right)\right)^{n}}{2}$ and

$$
\left|\delta_{n}\left(x_{i}\right)-\gamma_{n}\right|<\varepsilon_{m(n)} \text { for all }\left(x_{i}\right) \prec\left(y_{m(i)}\right)_{n}^{\infty} .
$$

Let $N_{0}=\left(\min \operatorname{supp} y_{m(i)}\right)_{i}=\left(n_{i}\right)_{i}$. Passing to a subset $N=\left(n_{k_{i}}\right)_{i}$, we may assume that for every $\alpha<\omega_{1}$, if $\left(n_{k_{i}}\right)_{i \in F} \in \mathcal{S}_{\alpha}$, then $\left(n_{k_{i}}\right)_{i \in F \backslash \min (F)} \in \mathcal{S}_{\alpha}(N)$. In particular $\left(k_{i}\right)_{i \in F \backslash \min (F)} \in \mathcal{S}_{\alpha}$. We shall prove that the subspace $Y=\overline{\operatorname{span}}\left\{y_{m\left(k_{i}\right)}\right\}$ is arbitrarily distortable. Let $y_{m\left(k_{i}\right)}^{*} \in B_{X}^{*}$ such that $y_{m\left(k_{i}\right)}^{*}\left(y_{m\left(k_{i}\right)}\right)=\left\|y_{m\left(k_{i}\right)}\right\|$ and range $\left(y_{m\left(k_{i}\right)}^{*}\right)=\operatorname{range}\left(y_{m\left(k_{i}\right)}\right)$ for every $i \in \mathbb{N}$. From the hypothesis we have that $X$ does not contain an $\ell_{1}^{\omega}$ spreading model. It follows that $\gamma_{\omega}=0$, for otherwise there exists $\left(x_{i}\right) \prec\left(y_{m\left(k_{i}\right)}\right)$ such that $\delta_{\omega}\left(x_{i}\right)>0$, and therefore $\left(x_{i}\right)$ would be an $\delta_{\omega}\left(x_{i}\right)-\ell_{1}^{\omega}$ spreading model. Since $\left(\gamma_{\alpha}\right)_{\alpha}$ is a continuous function of $\alpha$ it follows that $\gamma_{n} \searrow 0$. We set

$$
\begin{gathered}
A_{n}^{*}=\left\{x^{*}: x^{*}=\frac{\gamma_{n}}{2} \sum_{i \in F} x_{i}^{*}, x_{i}^{*} \in B_{Y^{*}},\right. \\
\left.y_{m\left(k_{n}\right)}^{*} \leq\left(x_{i}^{*}\right)_{i \in F} \text { is } \mathcal{S}_{n} \text {-admissible w.r.t. }\left(y_{m\left(k_{i}\right)}^{*}\right)_{i}\right\}, \\
A_{n}=\left\{y \in S(Y): y \text { is } \frac{1}{6} \text {-normed by } A_{n}^{*}\right\} .
\end{gathered}
$$

We observe that $A_{n}^{*} \subset B_{Y^{*}}$. Indeed, first we observe that if $\left(x_{i}\right) \prec\left(y_{m\left(k_{i}\right)}\right)_{n}^{\infty}$, then

$$
\frac{1}{2} \delta_{n}\left(x_{i}\right) \leq \delta_{n}\left(x_{i}\right)-\varepsilon_{m\left(k_{n}\right)}<\gamma_{n}<\delta_{n}\left(x_{i}\right)+\varepsilon_{m\left(k_{n}\right)} \leq 2 \delta_{n}\left(x_{i}\right),
$$

since $\delta_{n}\left(x_{i}\right) \geq\left(\delta_{1}\left(y_{i}\right)\right)^{n}>2 \varepsilon_{m\left(k_{n}\right)}$.

Let $x^{*}=\frac{\gamma_{n}}{2} \sum_{i \in F} x_{i}^{*} \in A_{n}^{*}$ and for $i \in F$ we set $E_{i}=\operatorname{range}\left(x_{i}^{*}\right)$. For every $y \in S_{Y}$ we have that

$$
\begin{aligned}
\left|x^{*}(y)\right| \leq \frac{\gamma_{n}}{2} \sum_{i \in F}\left|x_{i}^{*}(y)\right| & \leq \frac{\gamma_{n}}{2} \sum_{i \in F}\left\|y_{\mid \operatorname{range}\left(x_{i}^{*}\right)}\right\| \\
& \leq \delta_{n}\left(\left(y_{m\left(k_{i}\right)}\right)_{n}^{\infty}\right) \sum_{i \in F}\left\|E_{i} y\right\| \leq\|y\|,
\end{aligned}
$$

since $\left(E_{i}(y)\right)_{i \in F}$ is $\mathcal{S}_{n}$-admissible w.r.t. $\left(y_{m\left(k_{i}\right)}\right)_{n}^{\infty}$, and the basis is bimonotone. Also $A_{n}$ is an asymptotic set. Indeed, for any block sequence $\left(x_{i}\right) \prec\left(y_{m\left(k_{i}\right)}\right)_{n}^{\infty}$ from the stabilization of $\gamma_{n}$ we have that $\left|\delta_{n}\left(x_{i}\right)-\gamma_{n}\right|<\varepsilon_{m\left(k_{n}\right)}$, from which it follows that $\delta_{n}\left(x_{i}\right)<\gamma_{n}+\varepsilon_{m\left(k_{n}\right)}$. It follows from the definition of $\delta_{n}\left(x_{i}\right)$ that there exists a block sequence $\left(w_{i}\right)_{i \in F}$ of $\left(x_{i}\right)$ which is $\mathcal{S}_{n}$-admissible w.r.t. $\left(x_{i}\right)$ and therefore w.r.t. $\left(y_{m(i)}\right)$ as well, such that

$$
\delta_{n}\left(x_{i}\right) \sum_{i \in F}\left\|w_{i}\right\| \leq\left\|\sum_{i \in F} w_{i}\right\|<\left(\gamma_{n}+\varepsilon_{m\left(k_{n}\right)}\right) \sum_{i \in F}\left\|w_{i}\right\| .
$$


Let $x_{i}^{*} \in B_{Y^{*}}$ be such that $x_{i}^{*}\left(w_{i}\right)=\left\|w_{i}\right\|$ and $\operatorname{range}\left(x_{i}^{*}\right) \subset \operatorname{range}\left(w_{i}\right)$. We set $x^{*}=\frac{\gamma_{n}}{2} \sum_{i \in F} x_{i}^{*} \in A_{n}^{*}$, and $y=\frac{\sum_{i \in F} w_{i}}{\left\|\sum_{i \in F} w_{i}\right\|}$. Then we have that

$$
x^{*}(y)=\frac{\gamma_{n}}{2} \frac{\sum_{i \in F}\left\|w_{i}\right\|}{\left\|\sum_{i \in F} w_{i}\right\|} \geq \frac{\gamma_{n}}{2\left(\gamma_{n}+\varepsilon_{m\left(k_{n}\right)}\right)} \geq \frac{1}{6},
$$

since $\gamma_{n} \geq \delta_{n}\left(x_{i}\right)-\varepsilon_{m\left(k_{n}\right)}>\frac{\left(\delta_{1}\left(x_{i}\right)\right)^{n}}{2}>\varepsilon_{m\left(k_{n}\right)}$.

From the hypothesis we have that every normalized block sequence has a further block subsequence which is an $c-\ell_{1}^{k}$ spreading model, $k \in \mathbb{N}$. It follows that for every $\varepsilon>0$ every block sequence $\left(z_{i}\right) \prec\left(y_{m\left(k_{i}\right)}\right)$ contains $\frac{c}{2}$-normalized $(\varepsilon, n+1, n)$-s.c.c. Indeed let $0<\varepsilon<\frac{1}{2}$ and $\left(z_{i}\right)$ be an $c-\ell_{1}^{n+1}$ spreading model, and $\min \operatorname{supp} z_{i}=n_{l_{i}}$. Let $[n+1]_{1}^{L}=\sum_{i \in F} \alpha_{l_{i}} e_{n_{l_{i}}}$ be an $(n+1)$-average of a subset $L$ of $\left(n_{l_{i}}\right)$ with $l_{\min F}>3 / \varepsilon$. Then by the remark following Proposition 1.4 $\sum_{i \in F} \alpha_{l_{i}} e_{n_{l_{i}}}$ is an $(\varepsilon, n+1, n)$-basic s.c.c., $\left(n_{l_{i}}\right)_{i \in F} \in \mathcal{S}_{n+1}$, and by the properties of the set $N$, we have that $\left(l_{i}\right)_{i \in F \backslash \min (F)} \in \mathcal{S}_{n+1}$. Let $G=\left\{l_{i}: i \in F\right\}$ and set $x=\sum_{j \in G} \alpha_{j} z_{j}$. Then $x$ is an $(\varepsilon, n+1, n)$ s.c.c., and

$$
\|x\| \geq c \sum_{j \in G \backslash \min (G)} \alpha_{j} \geq c(1-\varepsilon),
$$

since $\left(z_{i}\right)$ is a $c-\ell_{1}^{n+1}$ spreading model. We set

$B_{n}=\left\{b: b\right.$ is an $\frac{c}{2}$-normalized $\left(\varepsilon_{m\left(k_{n}\right)}, n+1, n\right)$-s.c.c.

of a normalized block sequence of the basis of $Y$ \}.

From the above it follows that $B_{n}$ is an asymptotic set in $Y$. For every $b \in B_{n}$ we have

$$
\left|x^{*}(b)\right| \leq 3 \gamma_{n} \text { for every } x^{*} \in A_{n}^{*} .
$$

Indeed, let $\left(z_{i}\right)$ be a normalized block sequence of the basis of $Y, b=\sum_{i \in G} b_{i} z_{i} \in$ $B_{n}$, and $x^{*}=\gamma_{n} / 2 \sum_{k \in F} x_{k}^{*} \in A_{n}^{*}$. Set $I=\left\{i \in G: \operatorname{supp} z_{i} \cap \operatorname{supp} x_{k}^{*} \neq \emptyset\right.$ for at most one $k\}$, and $J=G \backslash I$. Also for every $i \in J$, let $K_{i}=\left\{k: \operatorname{supp} z_{i} \cap \operatorname{supp} x_{k}^{*} \neq \emptyset\right\}$. Then $\left(z_{i}\right)_{i \in J}$ is the union of at most two $\mathcal{S}_{n}$ admissible sets, hence

$$
\begin{aligned}
\frac{\gamma_{n}}{2} \sum_{k}\left|x_{k}^{*}\left(\sum_{i} b_{i} z_{i}\right)\right| & \leq \frac{\gamma_{n}}{2}\left(\sum_{i \in I} b_{i}\left\|z_{i}\right\|+\sum_{i \in J} b_{i} \sum_{k \in K_{i}}\left|x_{k}^{*}\left(z_{i}\right)\right|\right) \\
& \leq \frac{\gamma_{n}}{2}\left(1+\frac{4 \varepsilon_{m\left(k_{n}\right)}}{\gamma_{n}} \max _{i}\left\|z_{i}\right\|\right) \leq 3 \gamma_{n} .
\end{aligned}
$$

Combining (2.1) and (2.2), it follows that the equivalent norm $\mid\|y\|=\gamma_{n}\|y\|+$ $\sup \left\{x^{*}(y): x^{*} \in A_{n}^{*}\right\}$ gives a $\frac{1}{6 \gamma_{n}(1+6 / c)}$ distortion on $Y$. Since $\inf \left\{\gamma_{n}: n \in \mathbb{N}\right\}=0$ we have that $Y$ is arbitrarily distortable.

Remark. The arguments of Theorem 2.1 may give us another approach to the distortion of the space $X$, constructed by E.Odell and Th.Schlumprecht [13], which does not have any $\ell_{p}, 1 \leq p \leq \infty$, as a spreading model. Following [15], we have to consider the indices $\delta_{A_{n}}\left(\left(x_{i}\right)_{i}\right)$ for the families $A_{n}=\{F \subset \mathbb{N}: \# F \leq n\}$ and to prove a stabilization result for the sequence $\left(\delta_{A_{n}}\left(\left(x_{i}\right)_{i}\right)_{n}\right.$ for every block subspace. Since $\ell_{1}$ is finitely block representable in $X$, and $X$ does not contain $\ell_{1}$ spreading models, we may deduce that every subspace has an arbitrarily distortable subspace. 
Theorem 2.1] should be compared with the following result from [15]: Let $Y=$ $\left\langle\left(y_{i}\right)\right\rangle$ be a subspace of $X$, and $\left(\gamma_{\alpha}\right)_{\alpha}$ be stabilized by $\left(y_{i}\right)$. For $\alpha<\omega_{1}$ let $\hat{\gamma}_{\alpha}(Y)=\lim _{n}\left(\gamma_{\alpha \cdot n}(Y)\right)^{\frac{1}{n}}$. If $\lim _{n} \gamma_{\alpha \cdot n} \widehat{\gamma}_{\alpha}(Y)^{-n}=0$, then $Y$ contains an arbitrarily distortable subspace.

3.

Theorem 3.1. Let $X=T\left[\left(\mathcal{S}_{n}, \theta_{n}\right)_{n}\right]$ be such that $\theta_{n+m} \geq \theta_{n} \theta_{m}, \lim _{n} \theta_{n}=0$. Then the space $X$ does not contain an $\ell_{1}^{\omega 2}$ spreading model.

Proof. On the contrary assume that there exists a normalized block sequence $\left(x_{i}\right)_{i}$ which is an $2 c-\ell_{1}^{\omega 2}$ spreading model for some constant $2 c>0$. We shall prove that for every $n_{0} \in \mathbb{N}$ we have that $c \leq 10 \theta_{n_{0}}$, which yields that $c=0$.

By Proposition 1.1(b) choose $k(0) \in \mathbb{N}$ such that if $k(0) \leq F \in \mathcal{S}_{\omega}$, then $F \in \mathcal{S}_{\omega 2}$ and $k(n)$ such that if $k(n) \leq F \in \mathcal{S}_{\omega+n}$, then $F \in \mathcal{S}_{\omega 2}$. Without loss of generality we may assume that if $n \leq F \in \mathcal{S}_{n}$, then $F \in \mathcal{S}_{\omega}$.

Let $n_{0} \in \mathbb{N}$ and set $N_{0}=\left\{\min \operatorname{supp} x_{i}\right\}_{i \in \mathbb{N}}=\left(n_{i}\right)_{i}$. Define a sequence $\left(m_{i}\right)$ by the rule $m_{1}=n_{1}$ and $m_{i+1}=n_{m_{i}}$, and consider the subset $N=\left(n_{m_{i}}\right)_{i \in \mathbb{N}}$ of $N_{0}$. Passing to a further subset of $N$ and relabelling we may assume that $m_{\min N}>\max \left\{k(0), k\left(n_{0}\right)\right\}, \mathcal{S}_{n_{0}}\left[\mathcal{S}_{\omega}\right](N) \subset \mathcal{S}_{\omega+n_{0}}$ (by Proposition 1.1(c)), and moreover that the following holds:

$$
\forall \alpha<\omega_{1} \text {, if }\left(n_{m_{i}}\right)_{i \in F} \in \mathcal{S}_{\alpha} \text {, then }\left(n_{m_{i}}\right)_{i \in F \backslash \min (F)} \in \mathcal{S}_{\alpha}\left(N_{0}\right),
$$

hence $\left(m_{i}\right)_{i \in F \backslash \min (F)} \in \mathcal{S}_{\alpha}$ (by Proposition 1.1(a)).

Let $L_{0}=\left(n_{m_{i}}\right)_{M_{0}} \subset N$ be such that $m_{\min L_{0}}>\min \left\{n_{0}, \frac{3}{\theta_{n_{0}}^{2}}\right\}$. Let $n_{1}>n_{0}+10$. Passing to further subset $L_{1}=\left(n_{m_{i}}\right)_{M_{1}}$ of $L_{0}$ we may assume that $m_{\min L_{1}}>$ $\min \left\{n_{1}, \frac{3}{\theta_{n_{1}}^{2}}\right\}$.

Let $\left[n_{1}\right]_{1}^{L_{1}}=\sum_{j \in F_{1}} \alpha_{m_{j}} e_{n_{m_{j}}}$ be the first $n_{1}$-average of $L_{1}$, where $\left(e_{i}\right)$ denotes the unit vector basis of the space $X$. We have that $\left[n_{1}\right]_{1}^{L_{1}}$ is an $\left(\theta_{n_{1}}^{2}, n_{1}, n_{1}-1\right)$ basic s.c.c. The set $\left(n_{m_{j}}\right)_{j \in F_{1}} \in \mathcal{S}_{n_{1}}$ and therefore by the property of the set $N$ we have that $G_{1}=\left\{m_{i}: i \in F_{1} \backslash \min \left(F_{1}\right)\right\} \in \mathcal{S}_{n_{1}}$. We set $J_{1}=\left\{m_{i}: i \in F_{1}\right\}$, i.e. $J_{1}=G_{1} \cup\left\{m_{\min F_{1}}\right\}$. Then $\left[n_{1}\right]_{1}^{L_{1}}=\sum_{j \in J_{1}} \alpha_{j} e_{n_{j}}$.

Set $y_{1}=\sum_{j \in J_{1}} \alpha_{j} x_{j}$. Then $y_{1}$ is a $\left(\theta_{n_{1}}^{2}, n_{1}, n_{1}-1\right)$-s.c.c. of $\left(x_{i}\right)$ with $c \leq\left\|y_{1}\right\|$. Indeed, since $\left\{\min \operatorname{supp} x_{j}: j \in J_{1}\right\}=\left\{n_{m_{j}}: j \in F_{1}\right\}$ and $\left[n_{1}\right]_{1}^{L_{1}}$ is an $\left(\theta_{n_{1}}^{2}, n_{1}, n_{1}-1\right)$ basic s.c.c., we have that $y_{1}$ is an $\left(\theta_{n_{1}}^{2}, n_{1}, n_{1}-1\right)$ s.c.c. Also since $G_{1} \geq n_{1}$ and $G_{1} \in \mathcal{S}_{n_{1}}$, we have that $G_{1} \in \mathcal{S}_{\omega}$. Also from the choice of $L_{0}$, $G_{1}>k(0)$. Therefore $G_{1} \in \mathcal{S}_{\omega 2}$, and

$$
\left\|y_{1}\right\| \geq\left\|\sum_{j \in G_{1}} \alpha_{j} x_{j}\right\| \geq 2 c \sum_{j \in G_{1}} \alpha_{j} \geq 2 c \sum_{j \in F_{1} \backslash \min \left(F_{1}\right)} \alpha_{m_{j}} \geq c,
$$

since $\left(x_{i}\right)_{i}$ is a $2 c-\ell_{1}^{\omega 2}$ spreading model.

Assume that we have chosen $y_{1}<y_{2}<\ldots<y_{\ell}$ and $n_{1}<n_{2}<\ldots<n_{\ell}$ such that

(1) Each $y_{r}=\sum_{j \in J_{r}} \alpha_{j} x_{j}$ is a $c$-normalized $\left(\theta_{n_{r}}^{2}, n_{r}, n_{r}-1\right)$ s.c.c. of $\left(x_{i}\right)$, for $r=1, \ldots, \ell$.

(2) $n_{r}<J_{r} \backslash \min \left(J_{r}\right)=G_{r} \in \mathcal{S}_{n_{r}}$ for $r=1, \ldots, \ell$.

(3) $\left\|y_{r}\right\|_{\ell_{1}} \leq \frac{\theta_{n_{r}}}{\theta_{n_{r+1}}}$ for $r=1, \ldots, \ell-1$ where $\|\cdot\|_{\ell_{1}}$ denotes the norm of the space $\ell_{1}$. 
Then we choose $n_{\ell+1}$ such that $\left\|y_{\ell}\right\|_{\ell_{1}} \leq \frac{\theta_{n_{\ell}}}{\theta_{n_{\ell+1}}}$ and $2<\theta_{n_{\ell}} / \theta_{n_{\ell+1}}$. We also choose a subset $L_{\ell+1}=\left(n_{m_{i}}\right)_{i}$ of $L \backslash \bigcup_{r=1}^{\ell} \operatorname{supp}\left[n_{r}\right]_{1}^{L_{r}}$ such that $m_{\min L_{\ell+1}} \geq$ $\min \left\{n_{\ell+1}, \frac{3}{\theta_{n_{\ell+1}}^{2}}\right\}$.

Let $\left[n_{\ell+1}\right]_{1}^{L_{\ell+1}}=\sum_{j \in F_{\ell+1}} \alpha_{m_{j}} e_{n_{m_{j}}}$ be the first $n_{\ell+1}$-average of the set $L_{\ell+1}$. Then $\left[n_{\ell+1}\right]_{1}^{L_{\ell+1}}$ is $\left(\theta_{n_{\ell+1}}^{2}, n_{\ell+1}, n_{\ell+1}-1\right)$ basic s.c.c. Also $\left(n_{m_{j}}\right)_{j \in F_{\ell+1}} \in \mathcal{S}_{n_{\ell+1}}$ and therefore by the property of the set $N$ we have that the set $G_{\ell+1}=\left\{m_{j}: j \in\right.$ $\left.F_{\ell+1} \backslash \min \left(F_{\ell+1}\right)\right\} \in \mathcal{S}_{n_{\ell+1}}$. We set $J_{\ell+1}=\left\{m_{j}: j \in F_{\ell+1}\right\}$. By the choice of $L_{\ell+1}$, we have that $n_{\ell+1} \leq G_{\ell+1}$, and therefore $G_{\ell+1} \in \mathcal{S}_{\omega}$. Also we have that $k(0) \leq G_{\ell+1}$, so we deduce that $G_{\ell+1} \in \mathcal{S}_{\omega 2}$.

We set $y_{\ell+1}=\sum_{j \in J_{\ell+1}} \alpha_{j} x_{j}$. With the same arguments as for $y_{1}$ we have that $y_{\ell+1}$ is a $\left(\theta_{n_{\ell+1}}^{2}, n_{\ell+1}, n_{\ell+1}-1\right)$-s.c.c. of $\left(x_{i}\right)$ with $\left\|y_{\ell+1}\right\| \geq c$.

We continue in the same manner to produce a sequence $\left(y_{r}\right)_{r \in \mathbb{N}}$ satisfying properties (1)-(3) for all $r$.

Now let $M \subset\left\{\min \operatorname{supp} y_{i}: i \in \mathbb{N}\right\} \subset L$ and let $\left[n_{0}\right]_{1}^{M}=\sum_{j \in G_{0}} \gamma_{m_{j}} e_{n_{m_{j}}}$ be the first $n_{0}$ average of the set $M$, such that $\left[n_{0}\right]_{1}^{M}$ is $\left(\theta_{n_{0}}^{2}, n_{0}, n_{0}-1\right)$-basic s.c.c. Then we have that $\left[n_{0}\right]_{1}^{M}=\sum_{j \in G_{0}} \gamma_{j} e_{n_{j}}$, where $G=\left\{m_{j}: j \in G_{0}\right\}$, and $G \backslash \min (G) \in \mathcal{S}_{n_{0}}$. Also we have that $\bigcup_{G \backslash \min (G)} G_{j} \in \mathcal{S}_{n_{0}}\left[\mathcal{S}_{\omega}\right]$ and therefore

$$
\left\{n_{i}: i \in \bigcup_{G \backslash \min (G)} G_{j}\right\} \in \mathcal{S}_{n_{0}}\left[\mathcal{S}_{\omega}\right](N) \subset \mathcal{S}_{\omega+n_{0}},
$$

by the choice of the set $N$. From the definition of $m_{i+1}=n_{m_{i}}$ it follows that

$$
H=\bigcup_{G \backslash \min (G)}\left(G_{j} \backslash \min G_{j}\right) \in \mathcal{S}_{\omega+n_{0}},
$$

since the families $\left(\mathcal{S}_{\alpha}\right)_{\alpha}$ are spreading. Indeed, let $G_{j}=\left\{m_{r_{1}^{(j)}}<m_{r_{2}^{(j)}}<\right.$ $\left.\ldots<m_{r_{j}^{(j)}}\right\}$. Then for every $k \geq 2, m_{r_{k}^{(j)}}=n_{m_{r_{k}^{(j)}-1}} \geq n_{m_{r_{k-1}^{(j)}}}$ and since $\left(n_{i}\right)_{i \in \bigcup_{G \backslash \min (G)} G_{j}} \in \mathcal{S}_{\omega+n_{0}}$ we have (3.1). Also $k\left(n_{0}\right)<H$ and therefore $H$ belongs to $\mathcal{S}_{\omega 2}$.

Set $z=\sum_{j \in G} \gamma_{j} y_{j}=\sum_{j \in G} \gamma_{j} \sum_{i \in J_{j}} \alpha_{i} x_{i}$. Since we have assumed that $\left(x_{i}\right)$ is a $2 c-\ell_{1}^{\omega 2}$ spreading model, setting $z_{i}=\gamma_{j} \alpha_{i} x_{i}$, for every $i \in G_{j}$ and every $j \in G$, we have that

$$
\begin{aligned}
& \|z\| \geq\left\|\sum_{i \in \bigcup_{j \in G \backslash \min (G)} G_{j}} z_{i}\right\| \geq 2 c \sum_{j \in G \backslash \min (G)} \gamma_{j} \sum_{i \in G_{j} \backslash \min \left(G_{j}\right)} \beta_{i} \\
& \geq 2 c \sum_{j \in G \backslash \min (G)} \gamma_{j}\left(1-2 \theta_{n_{j}}^{2}\right) \geq 2 c\left(1-\theta_{n_{0}}^{2}\right)\left(1-2 \theta_{n_{1}}^{2}\right) \geq c .
\end{aligned}
$$

The vector $z$ is also an $\left(\theta_{n_{0}}^{2}, n_{0}\right)$-R.I.s.c.c. That is, $z$ is of the form $z=\sum_{j=1}^{k} \gamma_{j} y_{j}$ such that

(1) $y_{j}$ is a $c$-normalized $\left(\theta_{n_{j}}^{2}, n_{j}, n_{j}-1\right)$-s.c.c.

(2) $n_{0}+2<n_{1}<\ldots<n_{k}$, and $\left\{\min \operatorname{supp} y_{j}\right\}_{j} \in \mathcal{S}_{n_{0}}$.

(3) $\left\|y_{j}\right\|_{\ell_{1}} \leq \frac{\theta_{n_{j}}}{\theta_{n_{j+1}}}$ and $2<\theta_{n_{j}} / \theta_{n_{j+1}}$. 
For such vectors we have that there exists a constant $M \leq 10$ such that

$$
\|z\| \leq M \theta_{n_{0}} .
$$

We refer to [3] (Corollary 2.15), 4] (Proposition 1.15), and 9] for a proof of this estimation. Therefore we have that $c \leq 10 \theta_{n_{0}}$. Since $n_{0}$ was arbitrarily chosen we have the result.

Remark. Let $X=T\left[\left(\mathcal{S}_{\xi_{n}}, \theta_{n}\right)_{n}\right]$ be such that $\lim \xi_{n}=\xi$ is a limit ordinal. If the sequence $\left(\xi_{n}, \theta_{n}\right)_{n}$ is "appropriate" chosen, quoting the above arguments, for appropriate $\left(\theta_{n}^{2}, \xi_{n}, \zeta\right)$-s.c.c., we have that the space $X$ does not contain $\ell_{1}^{\xi 2}$ spreading models. We do not have a proof of inequality (3.2) for every sequence $\left(\xi_{n}\right) \nearrow \xi$. We refer to 8 for a proof of inequality (3.2) for the appropriate chosen sequence $\left(\xi_{n}, \theta_{n}\right)_{n}$.

The same arguments apply in the case of $p$-spaces, and yield that those spaces contain no $\ell_{1}^{2}$ spreading model. A space $X$ is said to be $p$-space if $X=T\left[\left(\mathcal{A}_{n}, \frac{1}{n^{1 / q_{n}}}\right)\right]$, where $\frac{1}{p_{n}}+\frac{1}{q_{n}}=1$ and $p_{n} \searrow p \in[1, \infty)$ and $\mathcal{A}_{n}=\{F \subset \mathbb{N}: \# F \leq n\}$ where the norm is defined similarly to the one in Definition 1.2 (b). We can also apply the above argument for $A_{k}\left[\mathcal{S}_{1}\right]$ sequences to produce R.I.s.c.c. of length $k$ and in this case we know that the norm is less than or equal to $M n_{k}^{1 / p_{k}}[12$. Let us recall that the existence of $\ell_{1}$ spreading models in Schlumprecht's space [16] has been established in [11.

\section{REFERENCES}

[1] D.E. Alspach and S.A. Argyros, Complexity of weakly null sequences, Dissertationes Mathematicae 321 (1992), 1-44. MR 93j:46014

[2] G. Androulakis and E. Odell, Distorting mixed Tsirelson spaces, Israel J. Math. 109 (1999), 125-149. MR 2000f:46012

[3] S.A. Argyros and I. Deliyanni, Examples of asymptotic $\ell_{1}$ Banach spaces, Trans. Amer. Math. Soc. 349 (1997), 973-995. MR 97f:46021

[4] S.A. Argyros, I. Deliyanni, D.N. Kutzarova and A. Manoussakis, Modified mixed Tsirelson spaces, Journal of Funct. Analysis 159(1998), 43-109. MR 2000j:46031.

[5] S.A. Argyros, I. Deliyanni, A. Manoussakis, Distortion and Spreading models in Modified mixed Tsirelson spaces, preprint.

[6] S.A. Argyros, I. Gasparis, Unconditional Structures of weakly null sequences, Trans. of AMS, 353 (2001), 2019-2058. MR 2002b:46012

[7] S.A. Argyros, S. Mercourakis, A. Tsarpalias, Convex unconditionality and summability of weakly null sequences, Israel J. Math. 107 (1998), 157-193. MR 99m:46021

[8] S.A. Argyros, A. Tolias, Methods in the Theory of Hereditarily Indecomposable Banach Spaces, preprint.

[9] I. Gasparis, A Continuum of totally incomparable Hereditarily Indecomposable Banach spaces, to appear in Studia Math.

[10] I. Gasparis, Strictly Singular non-compact operators on Hereditarily Indecomposable Banach spaces, to appear in Proc. Amer. Math. Soc.

[11] D.N. Kutzarova, P.K. Lin, Remarks about Schlumprecht space, Proc. Amer. Math. Soc. 128 (2000), 2059-2068. MR 2000m:46031

[12] A. Manoussakis, On the structure of a certain class of mixed Tsirelson spaces, Positivity 5 (2001), 193-238. MR 2002g:46021

[13] E. Odell and Th. Schlumprecht, On the richness of the set of p's in Krivine's theorem, Oper. Theory, Adv. Appl. 77 (1995), 177-198. MR 96i:46015

[14] E. Odell and Th. Schlumprecht, A problem on spreading models, Journal of Funct. Analysis 153 (1998), 249-261. MR 99c:46006

[15] E.Odell, N. Tomczak-Jaegermann, R.Wagner, Proximity to $\ell_{1}$ and distortion in asymptotic $\ell_{1}$ spaces, Journal of Funct. Analysis 150 (1997), 101-145. 
[16] Th. Schlumprecht, An arbitrarily distortable Banach space, Israel J. Math. 76 (1991), 81-95. MR 93h:46023

[17] B.Tsirelson, Not every Banach space contains $\ell_{p}$ or $c_{0}$, Funct. Anal. Appl. 8 (1974), 138-141.

Department of Sciences, Technical University of Crete, 73100 Chania, Greece

E-mail address: amanouss@science.tuc.gr 